\title{
Cucurbit[7]uril nanoencapsulation reduces the unpalatability of bitter phytochemicals
}

\author{
Zeba Manzer ${ }^{1}$, Tanoy Dutta ${ }^{2}$, Apurba Lal Koner², Aniruddha Mitra ${ }^{1,3}$ \\ ${ }^{1}$ School of Biological and Environmental Sciences, Shoolini University of Biotechnology and \\ Management Sciences, Solan, India. \\ ${ }^{2}$ Department of Chemistry, Indian Institute of Science Education and Research, Bhopal, India. \\ ${ }^{3}$ Department of Zoology, Sarojini Naidu College for Women, Kolkata, India.
}

\section{Supporting Information:}

\section{Experimental details:}

Chemicals used: Sucrose (AR, Loba), caffeine (AR, Loba), strychnine ( $\geq 98 \%$, Sigma), thiamine hydrochloride (Sigma), potassium ferricyanide (Sigma), blue dye - brilliant blue FCF (Vidhi Speciality Food Ingredients Ltd, Mumbai), red dye - amaranth (Vidhi Speciality Food Ingredients Ltd, Mumbai). Cucurbit[7]uril (CB7) was synthesized and purified according to previous reports ${ }^{17}$ and further characterized by ${ }^{1} \mathrm{H}$ NMR spectroscopy and mass spectrometry. The spectroscopic experiments were performed using Milli-Q water obtained from the Milli-Q grade water system from Merck (USA) with resistivity $18.2 \mathrm{M} \Omega \cdot \mathrm{cm}$ at $298 \mathrm{~K}$.

Flies used: Canton S strain of Drosophila melanogaster (obtained from the laboratory of Dr. N.G. Prasad at IISER Mohali) was used in this study. Flies were cultured in Drosophila culture medium (cornmeal, agar, yeast, and sucrose mixed with an ethanolic solution of p-hydroxy methyl benzoate and propionic acid) and were reared at room temperature (20 to $25^{\circ} \mathrm{C}$ ) under the natural light-dark cycle, at the Zoology laboratory of Shoolini University (30 86’ N, $77^{\circ} 12^{\prime}$ E) at Solan (Himachal Pradesh, India). Flies were starved for $24 \mathrm{~h}$ (with access to water, but no food) before using them in food choice assays.

Food choice assay: Twenty flies were anesthetized by cooling a vial containing flies over ice for 5$10 \mathrm{~min}$ and were introduced into a pair of plastic petri plates (65 mm diameter) where the lower plate had 8 droplets (each droplet $-2 \mu \mathrm{L}$ ) of one kind of solution (either $10 \mathrm{mM}$ caffeine/strychnine + $5 \mathrm{mM}$ sucrose or $10 \mathrm{mM}$ CB7 encapsulated caffeine/strychnine $+5 \mathrm{mM}$ sucrose) mixed with either red (2 mg/mL) or blue dye (1 mg/mL), and 8 droplets of another kind of solution (1 mM or 5 $\mathrm{mM}$ sucrose) mixed with the alternate dye (blue/red). The droplets were placed alternately (Figure 3a). The flies were allowed to revive, and then the setup was kept in a horizontal position in the dark for 2 hours at room temperature (so that flies cannot see the color of the liquid they are consuming). Then the petri plates were placed at $0^{\circ} \mathrm{C}$ inside a refrigerator for 30 to 40 min to kill 
the flies. A binocular stereo zoom microscope (Magnus MSZ-Bi) was used to check the color of their abdomens, and thereby the kind of solution consumed by each fly was verified (red abdomen fed on solution mixed with red dye, blue abdomen - fed on solution mixed with blue dye, purple abdomen - fed on both kinds of solutions, abdomen without any extra color - did not feed on any solution). A preference index for the solution mixed with red dye was calculated for each replicate by dividing the total number of flies with red + purple abdomens by the total number of flies with colored abdomens (red + blue + purple). ${ }^{32}$ Likewise, a preference index for the solution mixed with blue dye was also calculated.<smiles>Cc1ncc(C[n+]2csc(CCO)c2C)c(N)n1</smiles>

Thiamine<smiles>CC1=C(CCO)SC2=Nc3nc(C)ncc3CN21</smiles>

Thiochrome

Figure S1: Synthesis of thiochrome from thiamine using potassium ferricyanide.

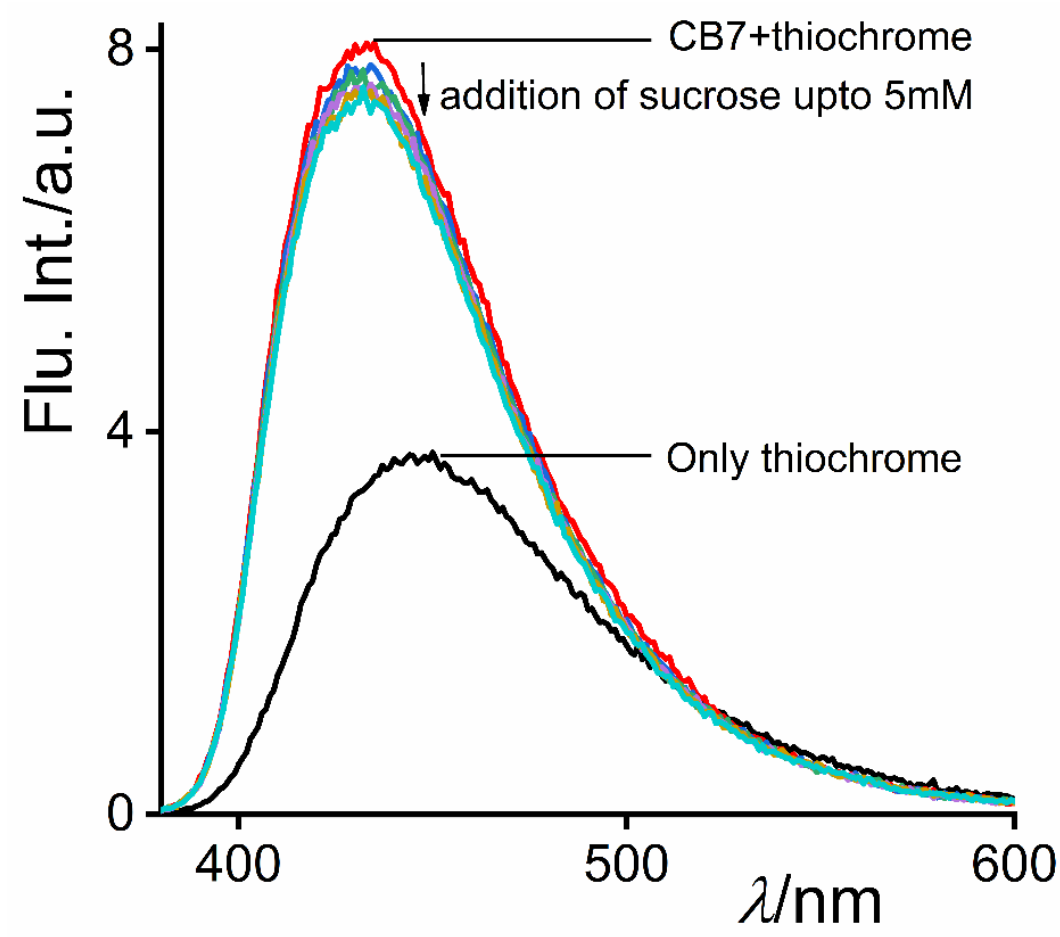

Figure S2: Plot showing fluorescence intensity of only thiochrome (5 $\mu \mathrm{M})$, the intensity of the CB7-thiochorme complex followed by the addition of sucrose (up to $5 \mathrm{mM}$ ) into it. 


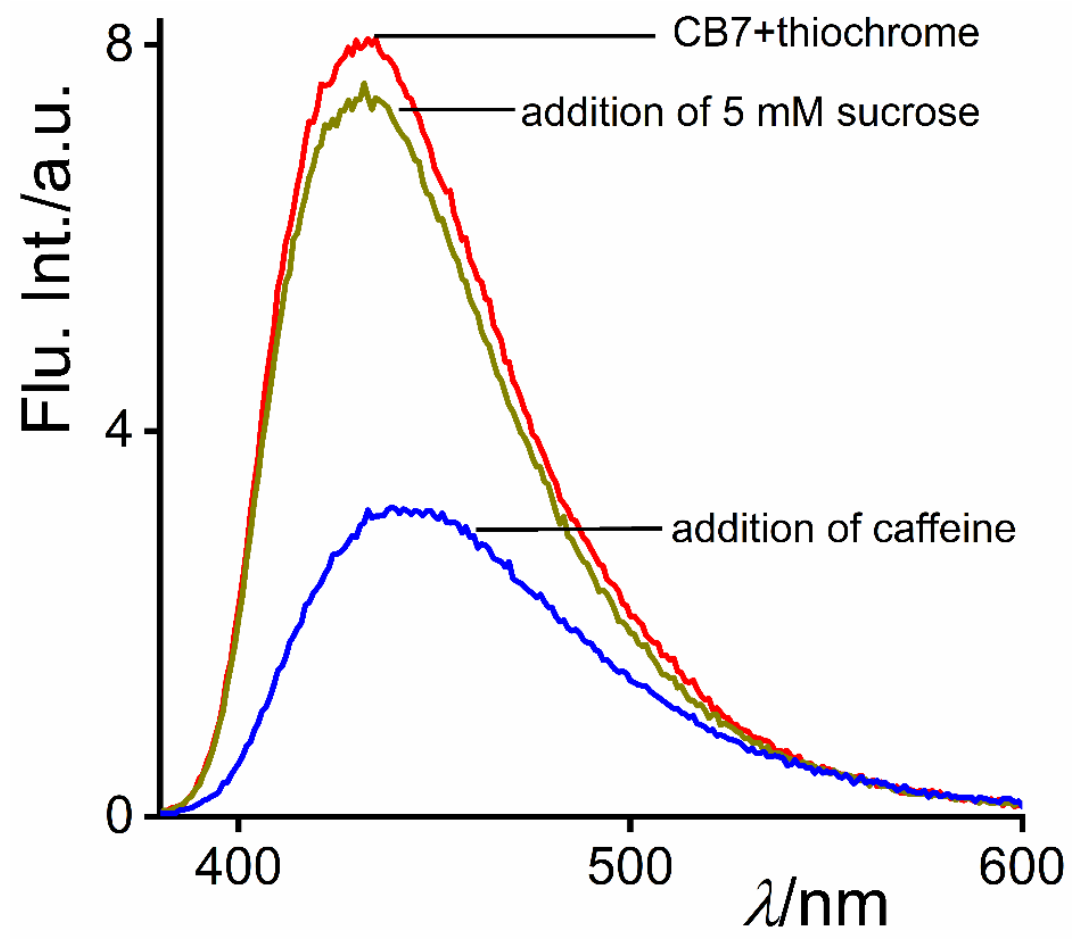

Figure S3: Fluorescence spectra of CB7-thiochorme complex (5 $\mu \mathrm{M}$ thiochrome and $0.5 \mathrm{mM}$ CB7), the addition of $5 \mathrm{mM}$ sucrose shows a negligible decrease in the intensity, and further addition of $0.5 \mathrm{mM}$ of caffeine replaces the bound thiochrome from CB7.

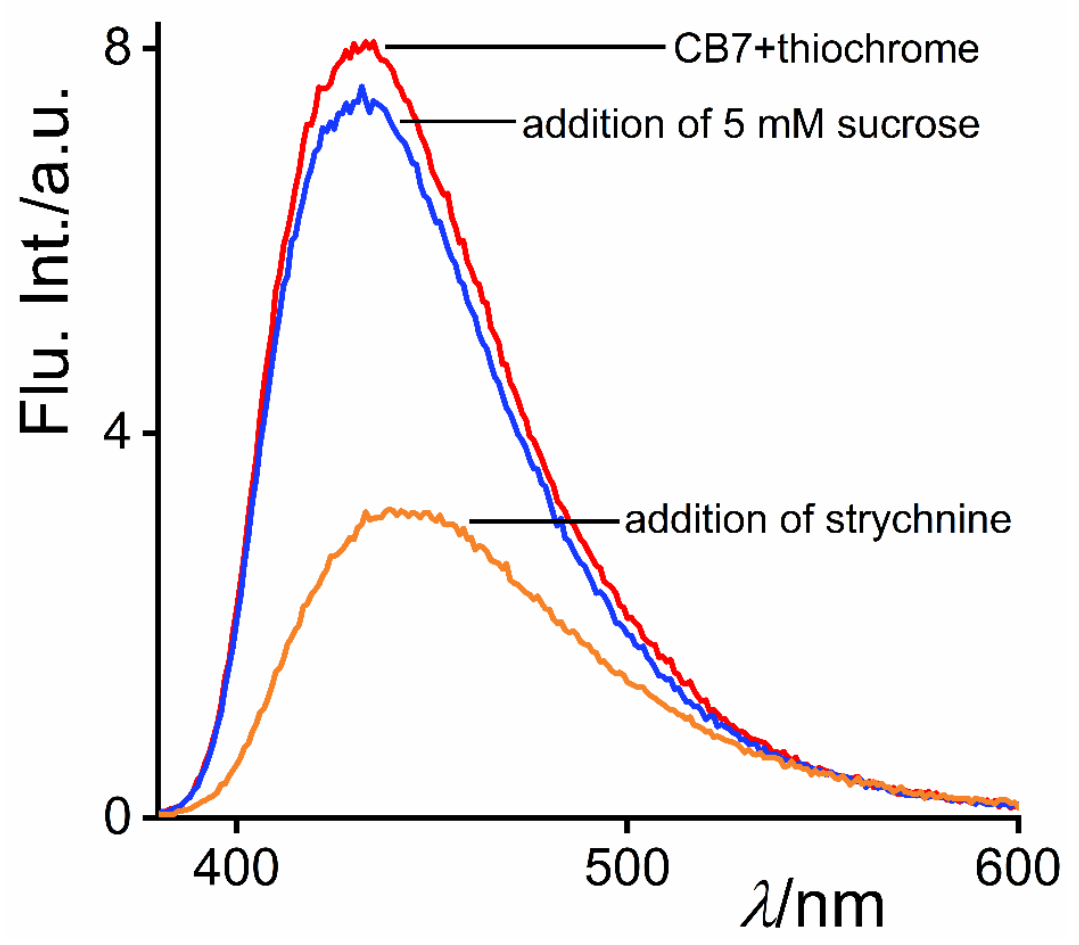

Figure S4: Fluorescence spectra of CB7-thiochorme complex (5 $\mu \mathrm{M}$ thiochrome and $0.5 \mathrm{mM}$ CB7), the addition of $5 \mathrm{mM}$ sucrose shows a negligible decrease in the intensity, and further addition of $0.5 \mathrm{mM}$ of strychnine replaces the bound thiochrome from CB7. 